\title{
LabVIEWBased Real Time GUI System Development for the studies of Ionospheric irregularities over near Equatorial region at Anantapur using Satellite Radio Beacon signals.
}

\author{
K. Jothi Babu' ${ }^{1}$, B. Rama Murthy², P. Thimmaiah ${ }^{3}$, K. Tanveer Alam ${ }^{3}$ \\ L. Suresh ${ }^{1}$, Mohammad. D. $\mathrm{V}^{4}$ \\ ${ }^{\text {I }}$ Al Musanna College of Technology, Al Musanna, Sultanate of Oman) \\ ${ }^{2}$ (Department of Instrumentation, Sri Krishnadevaraya University, Anantapur, A.P., INDIA) \\ ${ }^{3}$ (Department of Electronics, Sri Krishnadevaraya University, Anantapur, A.P., INDIA) \\ ${ }_{4}^{4}$ (Department of E\&I, AcharyaNagarjuna University, Guntur, A.P., INDIA)
}

\begin{abstract}
LabVIEW developed by National Instruments is a graphical programing tool based on the data flow. Due to its characteristics, the recent years it is an ideal tool for both design and implementation of embedded software. This paper presents the development of LabVIEW based Real time GUI system for the studies of variations of percentage of occurrence of VHF amplitude scintillation characteristics using radio beacon signals of $250.649 \mathrm{MHz}$ transmitted from FLEETSAT satellite positioned at $73^{\circ}$ E longitude received over near equatorial station Anantapur $\left(14.7^{\circ} \mathrm{N}, 77.7^{\circ} \mathrm{E}\right.$, Dip angle $\left.15.4^{\circ} \mathrm{N}\right)$. Already real time digital recording system isexisted, but the disadvantage with the system is inability of the graphical representation of amplitude scintillation patches. Hence, an attempt has been made to overcome this problem with the help of LabVIEW based GUI system for the amplitude scintillations suitable to have data compatibility with Excel and Origin graphical packages for further analysis of the Ionospheric irregularities Scintillation Index (SI),effect of Geomagnetic storms and power spectral variations with amplitude scintillations. The developed system is implemented and executed successfully.
\end{abstract}

Keywords:Ionosphere (Ionospheric Irregularities), Radio Science (Ionosphere Propagation, Space \& Satellite Communication), Magnetic Storm, LabVIEW (NI DAQ).

\section{Introduction}

LabVIEW is a graphical coding language and provides programming environment based on a data flow model. The main application of it was test, measurement used in industrial automation. At present there has been a tremendous growth in the embedded software systems market and LabVIEW plays a vital role in the reduction of hardware cost and need for fast potable solutions. National Instruments developed LabVIEW RT (Real Time) to answer these demands [1][2]. The main objective of the present work using the LabVIEW RT software and hardware environment for developing real time GUI system [3] to investigate the characteristics of VHF amplitude scintillations for the studies of Ionospheric irregularities over near equatorial region. The Radio wave communications are often subjected to marked changes in their performance owing to changes in the Ionosphere. The Ionospheric scintillations refer to random, rapid irregular and fast fluctuations in various parameters viz amplitude, phase as well as frequency shift of radio waves of VHF to L based transmissions from satellites received at the earth surface, whenever it is propagated through the medium which is non-homogenous in electron density(i.e. embedded with ionospheric irregularities [3][4].In addition the ionospheric scintillations are controlled by irregularity amplitude $(\Delta \mathrm{N} / \mathrm{N})$, background electron density $\mathrm{N}$ and its distribution in the ionosphere[5][6][7].

In the present work the LabVIEW based real time GUI system developed for recording the time amplitude scintillations and the data stored in a file further analysis with the graphical packages like Excel, Origin graphical packages etc. by replacing the existing real time data acquisition system developed in $\mathrm{C}$ language having disadvantages inthe graphical representation.

\section{Hardware Implementation}

The block diagram of the LabVIEW based GUI system for the studies Ionospheric irregularities is shown in figure 1 consists of the following units. They are

\subsection{Antenna System}

2.2. Frequency down counter

2.3. ICOMM communication receiver.

2.4. Digital data acquisition system. 


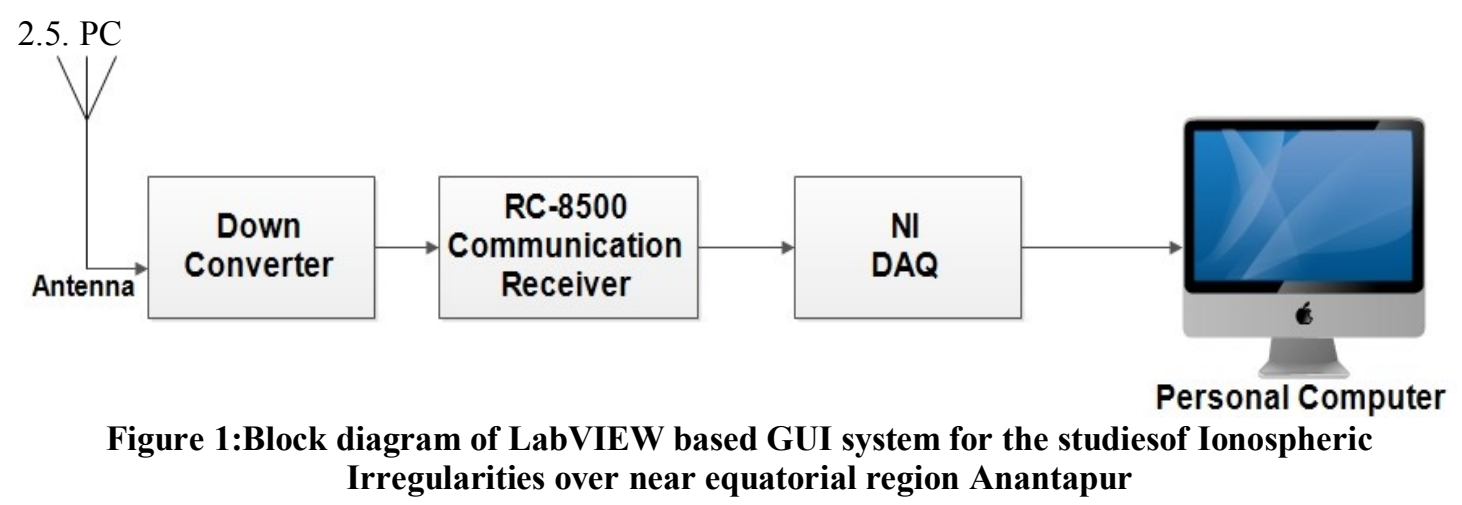

\subsection{Antenna System}

In general the type of antenna used in any communication network depends mainly on the frequency of operation. In the present study the frequency of the transmitted signal from the FLEETSAT satellite is 250.649 $\mathrm{MHz}$ and the free space attenuation (loss) suffered by the signal is large for the entire ray path from the satellite to ground and hence a high gain directional-antenna is essential at the front-end of the receiving system to obtain a high quality of scintillation data for studying the ionospheric irregularities [8].

A 12-element Yagi-Uda antenna is designed for this purpose with an operating centre frequency of $250.649 \mathrm{MHz}$, adopting the graphical design procedure described in ARRL Antenna Hand Book, (1976) for achieving a high gain with an optimum bandwidth and a narrow beam width, with increased directivity

\subsection{FrequencyDown Converter}

The radio frequency power radiated by the transmitter on-board the satellite is of the order of a few watts. Since the attenuation suffered by the signal in its propagation is very high, the strength of the signal received at the antenna is very weak of the order of a few micro volts, even with an antenna gain of $15 \mathrm{~dB}$ at the front end. Hence, in order to obtain high quality data with this low level signal, it is very essential to use a high gain and low noise pre-amplifier and frequency down converter. For this purpose a Frequency-down Converter Model No. 407G from the Vanguard Laboratories, USA is used.

\subsection{ICOM Communication Receiver (IC- R8500)}

In thepresent study the IC-R8500 issued which is the most sophisticated communication receiver from ICOM, JAPAN designed for a wide variety of applications covering HF, VHF and UHF bands all in one single, compact unit. The IC-R 8500 not only has normal tuning capability with the front panel, it can also be shifted quickly to a desired frequency by using the keyboard to program frequency data. In addition, tuning pitch can be selected between the following frequencies: $(10 \mathrm{~Hz}, 50 \mathrm{~Hz}, 100 \mathrm{~Hz}, 1 \mathrm{KHz}, 2.5 \mathrm{KHz}, 5 \mathrm{KHz}, 9 \mathrm{KHz}, 10 \mathrm{KHz}$, $12.5 \mathrm{KHz}, 20 \mathrm{KHz}, 25 \mathrm{KHz}, 100 \mathrm{KHz}$ and $1 \mathrm{MHz}$ ). With the help of tuning step switches and it can be also selected by using programmable tuning steps, which can be set between 0.5 and $199.5 \mathrm{KHz}$. The IC-R 8500 incorporates a total of six separate scanning functions for easy access to a wide range of frequencies [9].

\subsection{Data Acquisition System (NI USB-6009 DAQ)}

The LabVIEW based GUI system is developed using DAQ used is NI DAQ 6008/6009 from National Instruments. The DAQ hardware is what usually interfaces between the signal and a PC. It could be in the form of modules that can be connected to the computer's ports or cards connected to slots in the motherboard. DAQ cards often contain multiple components (multiplexer, ADC, DAC, TTL-IO, high speed timers, RAM). These are accessible via a bus by a microcontroller, which can run small programs. Many times reconfigurable logic is used to achieve high speed for specific tasks and digital signal processors are used after the data has been acquired to obtain some results. The fixed connection with the PC allows for comfortable compilation and debugging. Using an external housing a modular design with slots in a bus can grow with the needs of the user. The NI DAQ 6008/6009 is shown in figure 2 . The output of the RC- 8500 is given to the analog input of the DAQ which is connected with USB port of PC [10][11]. 


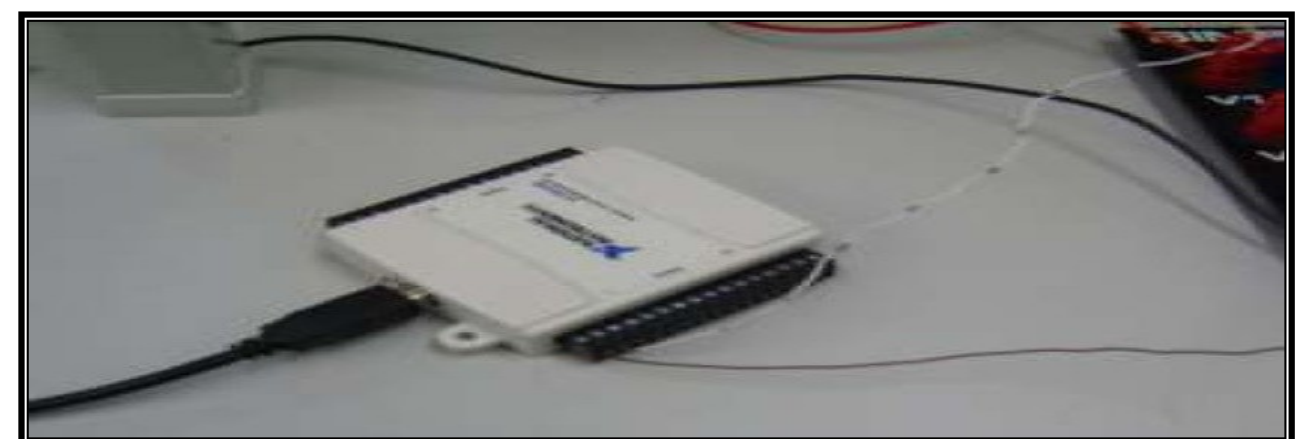

Fig: 2 NI-6009 DAQ

\subsection{Personal Computer}

In the present study, an Intel dual core based system (Dell) that consists of a system unit, 141 full function keyboard, super VGA colour monitor and a graphics adopter. The system consists of the Windows XP operating system with LabVIEW software is installed.

\section{Software Implementation}

Figure 3 shows the real time variations of amplitude scintillation on front window containing two charts indicators. The first window indicates record of the amplitude scintillation data for every one minute while the second window indicates the amplitude scintillation data for 12 hours from the start time of the system. This can be achieved with the implementation of LabVIEW using NI USB 6008 DAQ devicewith the following steps. Figure 4 shows total experimental set-up OF LabVIEW based GUI system forrecording the real time amplitude scintillations for the studies of Ionospheric irregularities [12].

- $\quad$ Configure the DAQ device in the Measurement \& Automation Explorer (MAX). Open LabVIEW.

- Create the DAQ Assistant on the block diagram window.

- Configure the measurement.

- Create the chart indicator on the front panel window.

- Wire the DAQ Assistant and chart indicator.

- Save and run the program.

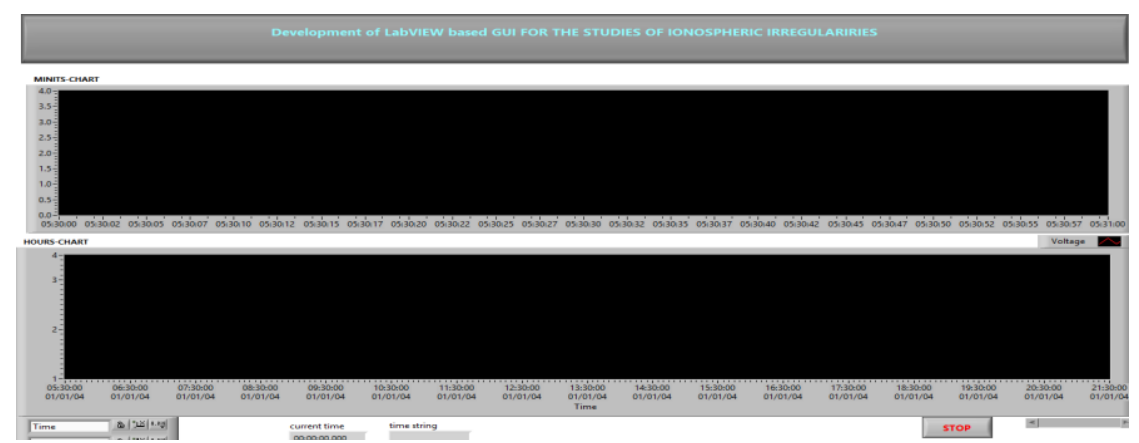

Figure 3:Windowof Front panel of the recording of Amplitude Scintillation Data.

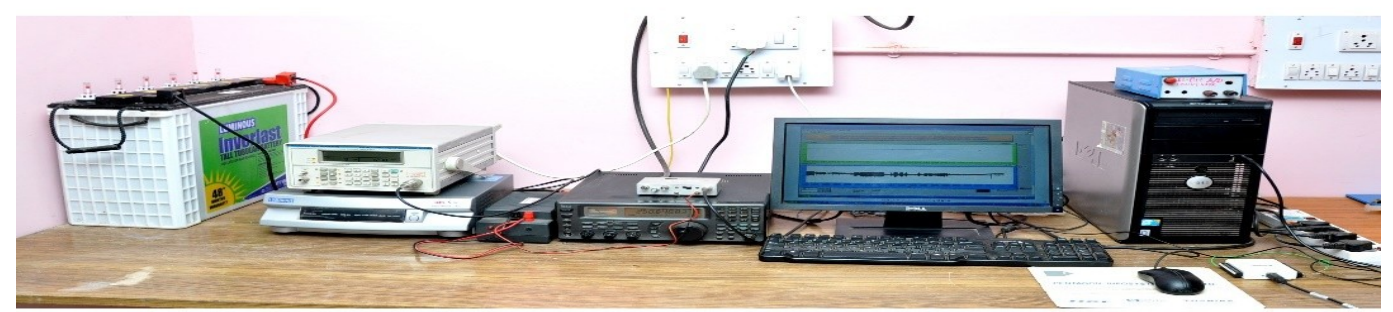

Figure 4: Experimental setup of the LabVIEW based GUI system for the studies of Ionosphericirregularities. 


\section{Creation Of Functional DiagramIn LabVIEW}

The DAQ Assistant is a graphical interface for interactively creating, editing and running NI-DAQ virtual channels and tasks. NI DAQ virtual channels consist of a physical channel on a DAQ device and the configuration information for this physical channel, such as input range and custom scaling should be provided by the user. A NIDAQ MX task is a collection of virtual channel, timing and triggering information, and other properties regarding the acquisition or generation. DAQ assistant provides an interactive guide to configuring, testing and acquiring measurement data. The DAQ assistant is completely menu driven and the user will encounter fewer errors [13].

When this express VI is placed on the block diagram, the DAQ assistant launches to create a new task. After creating the task, auser double click on the DAQ assistant express VI to edit that task

To launch the DAQ assistant, the following steps are to be followed

(i) Open LabVIEW and create a new VI. Switch to the block diagram.

(ii) DAQ Assistant express VI (A Sub VI designed to aid in common measurement tasks) is located in the input sub palette of the function palette as shown in fig 5

To create the task the steps to be followed are

(i) On the screen, select analoginput for your measurement type

(ii) Next select voltage

(iii) The next screen lets you to select the physical channel (or channels) for which you are creating this task.

(iv) Click finish to move on to the configuration stage.

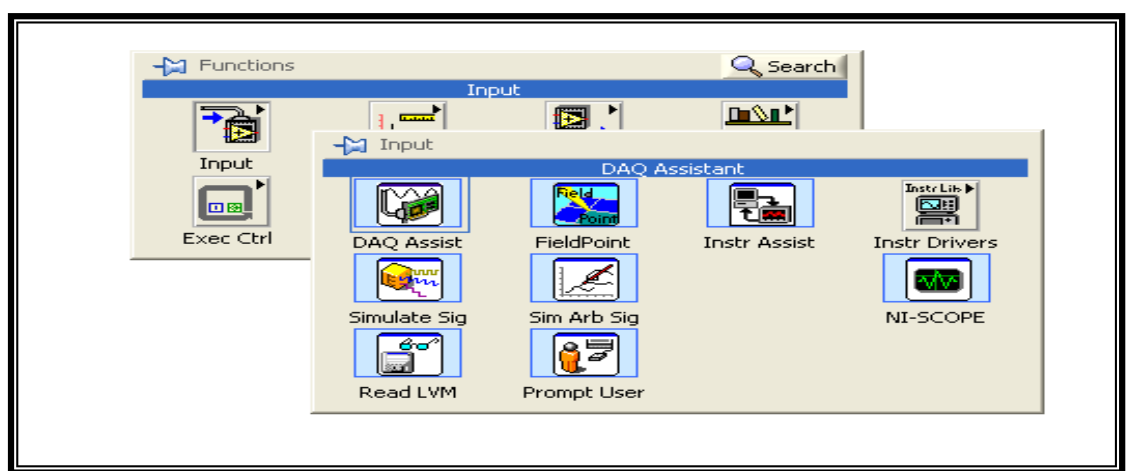

Figure 5: Launching of DAQ Assistant

Figure 6 shows the designing of complete functional block diagram of LabVIEW GUI system for recording the amplitude scintillations.

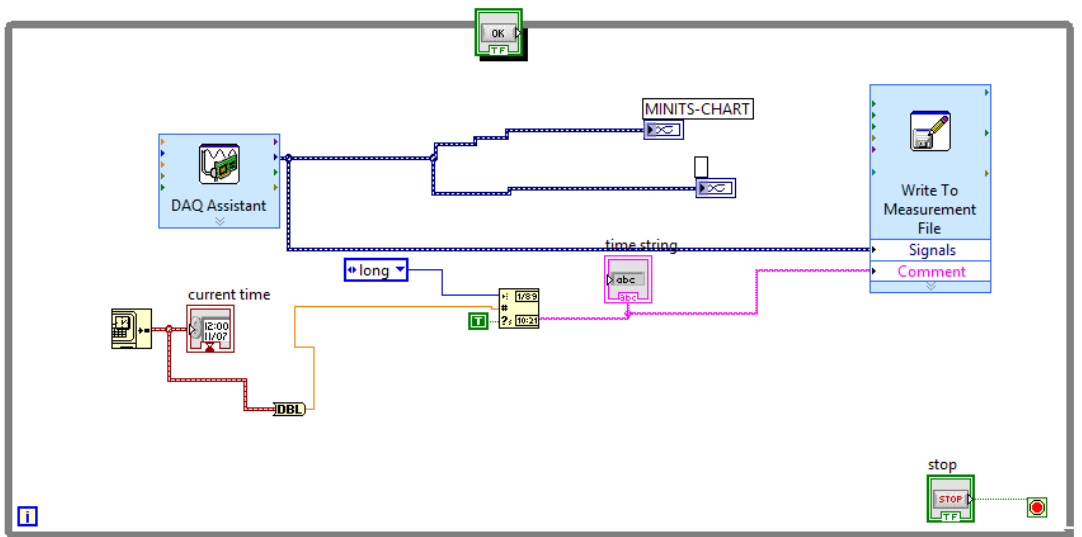

Figure 6:Functional Block of LabVIEW GUI System for recording Amplitude Scintillations

\section{Results And Discussion}

The developed hardware and software system is implemented and executedsuccessfully and the results are shown in figure 7 (a), 7(b) and 7(C) recorded in different dates and timings. The data is stored in a file inthe format of Real Time (HH:MM: SS.ms) in column 1 and amplitudeof scintillation (in volts) in column 2. The data is quite compatible with Excel and Origin graphical packages for further analysis like Scintillation Index (SI), effect of Geomagneticstorms [14] and power spectral variations [15] with amplitude scintillation characteristics for the studies of Ionospheric irregularities. The development of this LabVIEW GUI system is 
quite useful for the researchers doing their research investigations on upper atmospheric studies particularly to Ionosphere.

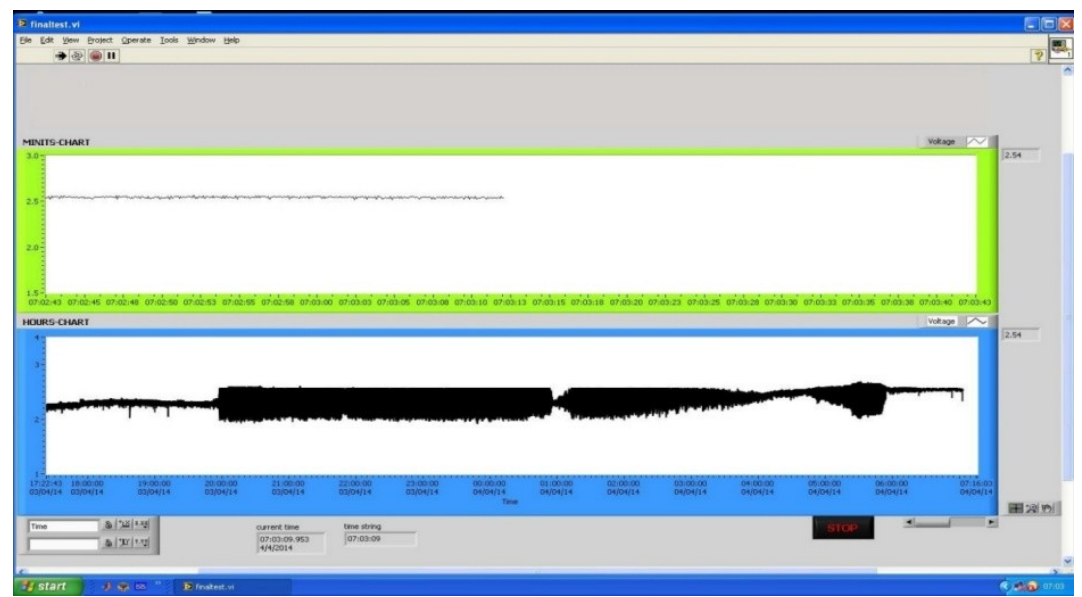

Figure 7(a) :Windowof Amplitude scintillation using LabVIEW GUI system

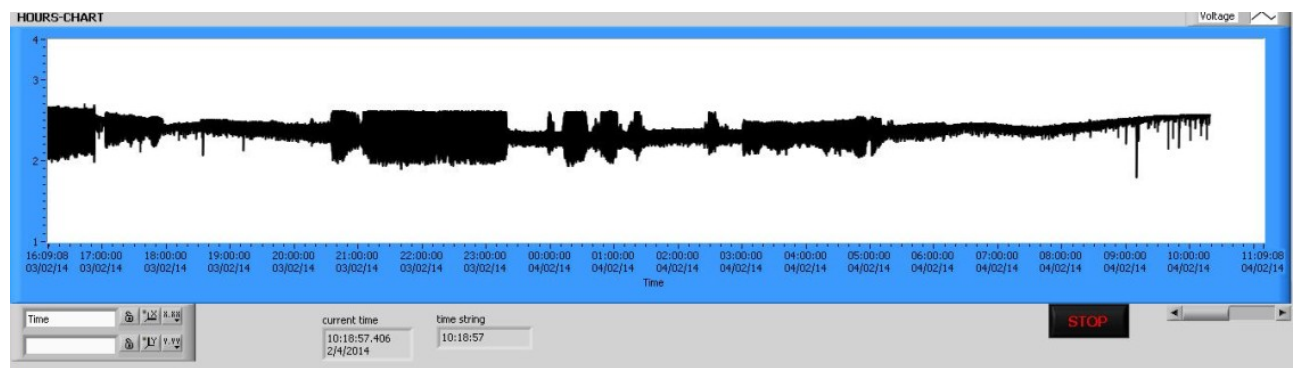

Figure 7(b): Window Night Time VHF scintillations occurrence on $23^{\text {rd }}$ January 2014

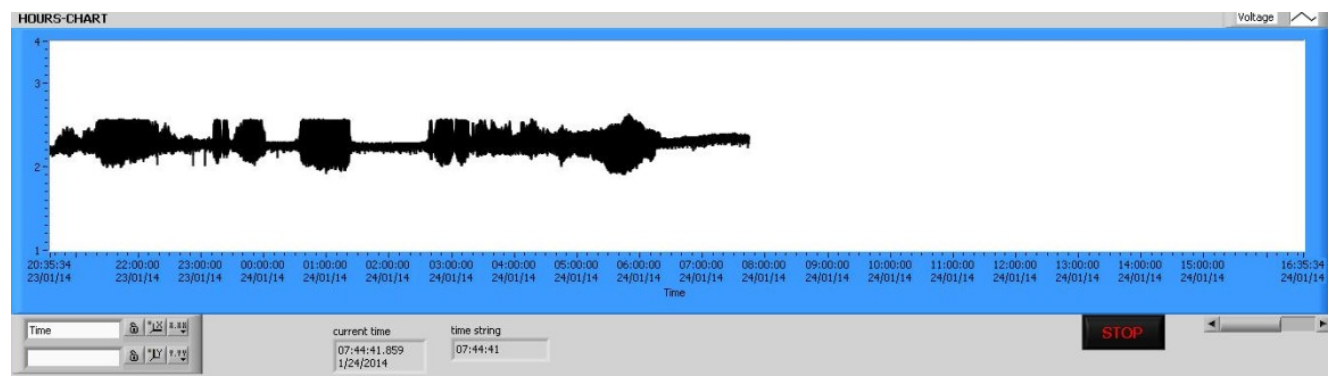

Figure 7(c): Window Night Time VHF scintillations occurrence on $3^{\text {rd }}$ February 2014

\section{References}

[1] Ram Rajagopal,SubramanianRamamoorthy,LotharWenzel,andHugoAndrade A Rapid Prototyping Tool for Embedded, Real-Time Hierarchical Control Systems, Hindawi Publishing Corporation, EURASIP Journal on Embedded Systems, Volume 2008, Article ID 162747,14 pages , doi:10.1155/2008/162747

[2] B V Sumangala\& K Bhargava Ram Advantages of LabVIEW over Embedded System in Home Automations International Conference on Advancement in Engineering Studies \& Technology, ISBN : 978-93-81693-72-8, 15th, JULY, 2012, Pondicherry.

[3] LabVIEW Graphical Programming by Gary W Johnson (TMH).

[4] Ram Rajagopal,SubramanianRamamoorthy,LotharWenzel,andHugoAndrade, Ram ajagopal,SubramanianRamamoorthy,LotharWenzel,andHugoAndrade, Rajagopal,SubramanianRamamoorthy,LotharWenzel,andHugoAndrade

[5] Aarons J, Whitney H E, Mackenzie E \&Basu S, Microwave equatorial scintillations intensity during solar maximum, Radio Sci(USA), 16 (1981) 939.

[6] Chakraborty S K, Das Gupta A, Ray S \& Banerjee S, Long term observations of VHF scintillations and total electron content neat the crest of the equatorial anomaly in the Indial longitude zone, Radio Sci (USA), 34 (1999) 241.

[7] Ray S, Paul A \& Das Gupta A, Equatorial scintillations in religion to the deveoplemnt of ionizationanonamy, Ann Geophys (Germany), 24 (2006) pp 1429-1442.

[8] P.Patel, K.Patel1, A.K.Singh1 and R.P.Singh, Simultaneous observation of VLF and VHF wave in the presence of ionospheric irregularities J. Ind. Geophys. Union (April 2010), Vol.14, No.2, pp.139-148

[9] S. Banola1, B. M. Pathan1, D. R. K. Rao1, and H. Chandra2, Spectral characteristics of scintillations producing ionospheric irregularities in the Indian region Earth Planets Space, 57, 47-59, 2005

[10] Eranna .U, Rama Murthy .B, Bhanu Prasad .K, Manjula .R "Study of Ionospheric Irregularities over near Equatorial Station Anantapur Using VHF Scintillations”, IETECH Journal of Communication Techniques, Vol.2, No.3. pp 157-163. 
[11] TiwariRajinder, Singn R.K, “A Real Time based Intelligent System Designed for the process Automation \& Control Applications. European Journal of Academic Essays 1(2) : 40-47, 2014.

[12] BogdanMihai. Virtual Signal Generator using the NI USB 6008 Data Acquisition devices Nonconventional Technologies Review 2013 Romanian Association of Nonconventional Technologies Romania, June, 2013

[13] David J. Ritter LabVIEW GUI: Essential Techniques, Volume 1

[14] Eranna .U, Rama Murthy .B, Bhanu Prasad .K, Manjula .R "Effect of Geomagnetic Storms on VHF Scintillations over near Equatorial station Anantapur", Ubiquitous Computing and Communication Journal,

[15] Rama Murthy .B, Eranna .U, Bhanu Prasad .K, Manjula .R "Power Spectral Studies of VHF Scintillations over near Equatorial station Anantapur", International Journal on Intelligent Electronic Systems, Vol.3., No.1, pp50-61. 2009.

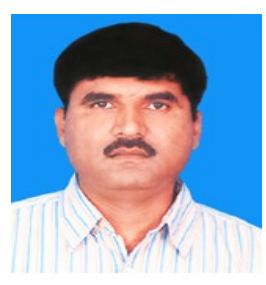

Mr. K. Jothibabu is presently working as a senior lecturer in the Department of Electrical Engineering,Al Musanna college of Technology, Sultanate of Oman. Now he is perusing his Ph.D in the Department of Electronics, Sri Krishnadevaraya University, Anantapur. His research interest areas of Communications and Data analysis, Industrial and process Engineering, Network security systems etc.

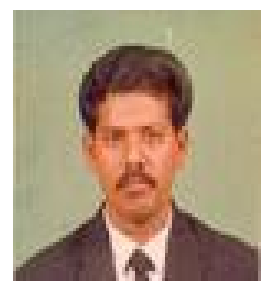

Dr.B.RamaMurthy is presently working as a Professor in the Department of Instrumentation \& USIC, Sri Krishnadevaraya University, Anantapur, Andhra Pradesh, India. He is having 21 years of Research \&Teaching experience. Under His guidance 8 Ph.D's\& 4 M.Phils are awarded. His areas of interest are Embedded Systems, Network and Mobile Communications, Network security systems, Industrial Automation and Bio-medical Instrumentation. 